\title{
METÁFORA DEL CONTRATO
}

\section{1. «Interference for other's own good»}

\author{
Chi consideri l'elemento \\ sociale altrimenti che \\ in modo simbolico, sbaglia. \\ Hugo von Hofmannsthal, \\ Buch der Freunde, trad. it., \\ Milán, 1980, 26.
}

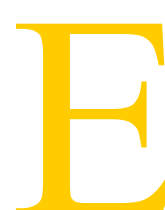

1 término paternalismo, en la tradición más antigua del lenguaje político, es utilizado para indicar una técnica de gobierno caracterizada por comportamientos autoritarios, aunque benévolos, de los gobernantes en sus relaciones con los gobernados. Una técnica que, siendo un modelo de antropología política tradicionales. complejo y articulado, no se agota dentro de los sistemas de sociedades

El horizonte semántico de conceptos como autoritarismo y benevolencia es naturalmente lo bastante amplio para hacer oscilar el término paternalismo tanto en sus determinaciones históricas como en las estructurales, y de ese modo poder ser empleado ya sea en su relación al soberano iluminado del setecientos europeo o en relación con el asistencialismo del welfare. Por otra parte, es suficiente el término «benévolo» para sobrecargar al paternalismo con un plus de ideología que, aunque observado ya por la teoría clásica, ha terminado por hacer aumentar en la reflexión politológica contemporánea los juegos de imputación, contribuyendo a enriquecer aquella semántica ya compleja de por sí. Desde algunos puntos de vista, se puede decir que de hecho la pérdida de consistencia del concepto de paternalismo en este siglo está ligada a una mengua ideológica de los términos tradicionales de lo ético-político. Es así que el paternalismo comienza a figurar en el léxico político en parejas antagónicas que lo contraponen con autonomía, autodeterminación, igualdad; en una palabra: con democracia. Parecería así que, después de la aparición de teorías críticas más o menos libertarias que habían animado el debate politológico hace algunas décadas, el término paternalismo se haya revelado en todo su significado negativo; como si la modernidad estuviera marcada por un camino irreversible «hacia una sociedad sin padre» y que la democracia, 
consiguientemente, fuera el elemento verdaderamente «moderno» -por lo tanto vencedor- de esta pareja conceptual antitética.

A pesar de que tal mengua ideológica parecía definitiva, a partir de los años 70 en adelante, particularmente bajo la influencia de la estadounidense Philosophy and Public Affairs y a la sombra muchas veces ingenua del neocontractualismo, este «antiguo nombre» ha reencontrado un éxito inesperado precisamente en el ámbito de una filosofía jurídico-política que, antes que otras, había ya problematizado el tema de la crisis del Estado social. Surge entonces un problema no secundario de semántica histórica que repropone el nuevo éxito de un concepto antiguo. Es desde esta perspectiva particular de la oposición paternalismo-democracia que se puede intentar una reconstrucción de nuestro tema que no resulte banal.

Hay que precisar sobre todo que aquí utilizamos la expresión «semántica histórico-política» en el sentido atribuido por R. Koselleck $(1986,185)$ en sus numerosos trabajos en torno a la Geschichtliche Grundbegriffe: como el conjunto de posibilidades lingüísticas que se transforman desplazando «espacios de experiencia» y abriendo nuevos «horizontes de expectativas»; y es sabido que, desde este punto de vista, la antinomia metodológica que subsiste entre la irrepetibilidad histórica y la repetibilidad estructural de las figuras lingüísticas individualiza una «historia» que nunca es idéntica a su expresión lingüística. Se manifiesta, en otras palabras, una elección entre la contingencia y las posibilidades de sentido de las expresiones lingüísticas, respecto de las cuales es necesario ahondar. Hay que señalar que las parejas antitéticas de conceptos son grandes contenedores de sedimentaciones de «sentido» histórico. "Si se examina su estructura, las parejas de conceptos -escribe Koselleck- pueden ser separadas de su origen anterior y de aquello que forjó su contexto concreto: son históricamente transferibles. Es precisamente esto lo que permite que exista una historia de la influencia ejercida por los conceptos; es justo aquí donde arraigan los hallazgos estructurales, los cuales demuestran como algunas redes de la experiencia pueden ser utilizadas repetidamente y permiten la aprehensión de ciertas analogías... Los espacios de experiencia se desplazan y se abren nuevos horizontes de expectativas.»

Es más bien en las transformaciones semánticas del paternalismo y de su oposición a la democracia que emergen representaciones fundamentales de la justificación del poder de interferir en la esfera de otros. Un dato interesante es que en el redescubrimiento del paternalismo producido en la filosofía «pública» 
de los años 70, el deslizamiento de los sistemas ético-políticos al sistema jurídico ha signado contemporáneamente su confinamiento a una dimensión de micro-relaciones sociales de naturaleza «privatista», lo cual tiene como efecto no secundario el de vaciar de contenidos quizás solamente «simbólicos» no tanto al paternalismo mismo como al de democracia. Es decir, que en la diferenciación y en la particularización del paternalismo jurídico hay algo de más y de mayor importancia que una pura y simple técnica jurídica, y del mismo modo, aunque no exclusivamente, cuando éste viene justificado por preocupaciones de distributive Justice. Desde una perspectiva sustancialmente consensualista del intercambio social, el paternalismo jurídico se manifiesta en el reconocimiento de la legitimidad del principio milliano -«one very simple principle»- del self protection en el poder de interferir en la esfera de libertad de otro individuo. La definición adoptada por G. Dworkin $(1983,19)$, es la siguiente: «Por paternalismo se entiende una interferencia en la libertad de acción de otra persona, justificada exclusivamente por el bienestar, necesidades, intereses, felicidad, valores, de la persona coercionada.» Si self protection significa entonces tanto evitar daños al individuo como a la sociedad; es en cambio más difícil establecer cuando se está frente a una tutela de la felicidad, de los valores y de los intereses de la persona. Basta para esto recordar, cosa que no siempre es hecha, la refinada elaboración iheriniana sobre el concepto de interés y de lucha individual por el derecho para tener un cuadro de referencia sobre la ambigüedad semántica de estas cláusulas generales. Aun aceptando, por consiguiente, que el principio adoptado no es el único (one) ni very simple, todo el esfuerzo «garantista» de esta Philosophy and Public Affairs se concentra tanto en la tipología de las interferencias justificadas o justificables, como en la elaboración de criterios guías de las potestades delegadas. De ello resulta una taxonomía divertida y en cualquier caso útil que ahonda en la vieja dogmática de la patria potestad, así como en las situaciones más nuevas y recientes sugeridas por los casos judiciales hard o por discusiones públicas sobre particulares controversias legislativas. Los ejemplos, aunque no siempre convincentes, son por lo menos sugestivos: desde el uso del casco para los motociclistas pasando por la prohibición del suicidio, hasta llegar al recurso de prácticas médicas contra la voluntad o las opciones religiosas de los pacientes (el caso reciente de la transfusión de sangre respecto de los testigos de Jehová) y así también en otros casos. Y resulta todavía útil la tentativa de clasificación sugerida respecto al paternalismo consensual (el caso de Ulises que se deja atar por sus compañeros 
para resistir el canto de las sirenas) o más precisamente voluntarista y al paternalismo conflictivista, como en el caso de recursos pedagógicos, educativos o terapéuticos que van contra la determinación individual de los pacientes; incluso se habla de paternalismo activo y omisivo (el caso de las mentiras piadosas o de «finalidad bondadosa»). Pero no cabe duda que estas tipologías se elaboran sobre una estructura tautológica de la definición del fenómeno.

Tautología, por cierto, que no se elimina cuando, yendo al fundamento del poder de interferir, la imaginación ética (V. Camps, 1983) de los neo contractualistas sugiere un presupuesto de consenso contra la debilidad de los individuos desfavorecidos. La tautología radica en el hecho que un acuerdo originario presupone no tanto una condición de igualdad, cuanto la universalidad de las decisiones. No es casualidad que, hablando de las desigualdades y de los problemas relativos al fairness, J. Rawls (1971, 182-214) escriba que los principios del paternalismo sean aquellos «que las partes reconocerían en la posición original contra la debilidad y las enfermedades de su razón y de su voluntad». Lo cual quiere decir, obviamente, poner otra vez la discusión sobre los principios contractuales de fondo, en los que se manifiestan problemas nodales (se podría decir kantianos) que se vinculan con la representación de la desigualdad y de los remedios «consensuales» para las condiciones de desventaja. Y en relación con esto, al carácter individual, podemos decir atómico, «micro-físico» de las condiciones de desigualdad; y no por último, al problema de la dimensión universal de las decisiones representativas; o bien al carácter de condición necesaria, pero no suficiente, de la representación para los fines de la legitimidad.

Como se observa, no obstante la naivetè de fondo de catálogos y decálogos de este paternalismo jurídico, la discusión no puede evitar los puntos nodales de la teoría política moderna. A cada instante, a pesar de su impostación, emergen temas más complejos de aquellos que esta filosofía del paternalismo jurídico no quería afrontar; temas que coinciden con los «nombres» que, sin ser nuevos, presiden las transformaciones del universo político moderno.

\section{El amor por sí}

Simetría-disimetría, igualdad-desigualdad, medida-desmesura, poder de interferencia-necesidad, privilegio-desventaja, constituyen parejas antagónicas de conceptos que individualizan el 
juego de la relación paternalista. Si se observa un poco más allá de la reducción privatista -aunque importante- y se analiza aquella micro-física de las desigualdades como un fenómeno colectivo de gran amplitud que se descarga sobre amplias franjas de población y que se reproduce en la dimensión internacional también en relaciones entre pueblos, resulta difícil separar la forma jurídica del paternalismo de su matriz estructuralmente política. Por lo demás es suficiente observar cómo, sobre todo en Europa y a partir ya desde muchas décadas, también dentro de la forma jurídica se ha impuesto históricamente un proyecto de mayor protección para categorías enteras de población desfavorecida. Sería necesario hablar más ampliamente y con mayor profundidad, mas puede aquí únicamente recordarse la experiencia italiana del «derecho desigual» que ha asumido, en el marco de un paradigma estrechamente jurídico, el problema de la subjetividad débil. Más allá de los resultados, generalmente opuestos a las intuiciones manifestadas en el terreno técnico-jurídico y en el político, aquella experiencia había asumido seriamente los problemas emergentes que esa «filosofía pública» ha relativizado quizás demasiado. Llevar otra vez el tema de la reflexión a argumentos tales como la representación, la universalidad de los intereses y las formas de la desigualdad, significa no solamente colocar de nuevo el concepto de política dentro de esta reducción política, sino también otorgarle repetidamente la complejidad necesaria.

Se podría profundizar en la literatura más clásica, desde los orígenes de la formación del concepto de lo político, para reencontrar el paternalismo como metáfora de la forma de gobierno de la polis. Creo que el ejemplo más evidente de «paternalismo puro» (G. Dworkin, 1983; R. Sartorius, 1983; E. Diciotti, 1986, 557) es el ofrecido por Platón en La República cuando hablando de la virtud de los gobernantes atribuye a ellos la capacidad de convencer al pueblo recurriendo también a «mentiras nobles» -se trataba en particular de la fábula fenicia relativa a la diversa «composición» de los estratos de la población: los guerreros, los guardianes, los campesinos-. Este es un ejemplo de paternalismo propiamente dicho porque construye una relación política sobre la base de la disimetría «cultural»y sobre todo para el caso de la mentira instrumental para el bienestar, para la felicidad, para los intereses del pueblo. Así, paradójicamente podríamos decir que poco importa que el argumento del bienestar de los gobernados sirviese para «justificar» la mentira y que entonces pudiese ser tachado como ideológico; el punto relevante es que dentro de la elaboración más robusta de la teoría de la política 
figura casi como elemento estructural el argumento paternalista. Por esto, tanto su supervivencia como su transformación se convierten en temas de semántica histórico-política.

La identificación de paternalismo y virtud clásica de los gobernantes (phronesis) tiene un sentido particular (una «semántica» histórica) en una polis (R. Havelock, 1979) que posee un nivel de diferenciación sólo segmentaria, en la cual las disimetrías incorporadas en la población constituyen la estructura misma del universum de la sociedad. La repetición de los sistemas segmentados hacen que no existan diferencias entre política, economía y sociedad (K. Planyi, 1974). Lo político no solamente representa por entero a la sociedad, sino que es el speculum. Será necesario esperar que en el ámbito del desarrollo histórico tengan lugar procesos más complejos de diferenciación entre los sistemas de la sociedad, para que las disimetrías cambien de signo y se elabore una semántica más específica en el plano de las relaciones políticas y de las formas representativas de la soberanía.

Desde la constitución del mito de la polis y siguiendo su historia y sus destinos, la idea del paternalismo aparece estrechamente vinculada con la estructura de la legitimación y con los fundamentos de la representación del «soberano». El largo proceso de secularización que ataca «el cuerpo» del rey sigue efectivamente la búsqueda de una nueva justificación de los fundamentos de la representación. La comunicación política, no ya referida a ordenamientos y a orientaciones de valores válidos desde siempre, resulta inmersa en un circuito dominado por la inestabilidad. La diferenciación interna en la sociedad, la diseminación de las diversidades de base económica, étnica, religiosa (el multi-versum) desarrollan progresivas necesidades de «uniformidad». Entre soberano y súbdito se instaura un juego complicado de comunicación política que pone en discusión, de modo totalmente particular, la distinción no igual de poder entre individuo y absolutismo. Se trata por tanto de ese particular juego manierista (R. Schnur, 1979) que ya a partir del siglo XVI introduce un nuevo antagonismo, el cual es, en realidad, un juego relacional muy complejo entre conformidad y diversidad, entre individuo y sociedad.

En la convincente reconstrucción que hace R. Schnur es precisamente la representación de la conformidad de los individuos a las reglas de la soberanía la que da el máximo de distancia entre la elaboración subjetiva del individualismo (no únicamente posesivo) y la regla colectiva de la obediencia. De este modo, se comienza a introducir una diversa semántica de la obediencia, en 
la cual la adhesión pública deja espacios a la libertad de comportamientos privados.

Pueden ser indicadores importantes el modo en el cual comienza a diferenciarse lo escrito ligado a las reglas de la comunicación pública con la conversación privada; la misma canonización del comportamiento del hombre de la corte que pre-supone una deformidad de actitudes; el énfasis sobre la conjura de palacio sobre la cual la literatura es riquísima: La interacción entre individuos comienza entonces de manera inequívoca a acrecentar la complejidad de las relaciones sociales; en pocas palabras: la diversidad de la sociedad y su articulación respecto a una política que uniforma de manera absolutamente artificial, hacen que los individuos se puedan representar como superiores a las relaciones políticas en las cuales viven. El mecanismo de auto-descripción de los individuos respecto a sus ambientes, más que ser importante para los fines de una teoría del individualismo es aquí relevante para el modo en el cual evidencia una fuerte crisis de la soberanía como representativa del todo, cuya regla es universal y en relación a la cual la obediencia se considera natural. Existen ya testimonios de una específica elaboración del concepto de secreto (R. Koselleck, 1979) y ello viene dado ya por el perfecto conocimiento del manierista político de un art of writing para huir de la persecution (L. Strauss, 1952), ya con el uso refinado de una sprachliches Doppelleben, o sea, de una continua disimulación lingüística. En el ambiguo código de la comunicación, escribe Schnur, «quien es consciente de pensar contra lo conforme, en tiempo de forzado conformismo (subrayado nuestro), usa valerse de determinados artificios para hacer que las propias ideas, una vez hechas públicas, no choquen con demasiada facilidad con la censura de los conformistas»».

Para llegar a esto, el «manierista» debe haber elaborado estrategias de representación del sí en relación a los diversos sistemas en los cuales opera y con los cuales interactúa (N. Luhmann, 1983 y 1985) estructurando sus límites. Su auto-observación funciona también como control de los procedimientos mediante los cuales funcionan los sistemas, particularmente el de la ética y el de la política. En la elaboración específica de un individuo escindido entre lo público y lo privado (Montaigne) se manifiesta la verificación del fin de un modelo de comunicación política y al mismo tiempo la expansión de una nueva relación con la soberanía. La conciencia de la conformidad es la primera gran Ausdifferenzierung entre ciudadanos y soberano, entre poder y política; paradójicamente, el manierista que acepta públicamente 
tal conformidad, la tolera; tolera la soberanía habiendo reconocido sus límites.

El soberano no puede mandar a las conciencias, sugiere el manierista, no tanto porque ha elaborado ya un concepto de intangibilidad de las esferas subjetivas (por esto necesitará todavía esperar), sino porque ahora está conociendo que el lugar del rey no es la sociedad entera. Se ha interrumpido la natural armonía entre la virtud, la felicidad y la ciudad; en el resquebrajamiento del mito de la ciudad el soberano no tiene más en sus manos el acontecer histórico como un cetro, para recurrir a la imagen de W. Benjamin. Cambia profundamente también la iconografía que acompaña y reconoce la armonía de la polis; en la fractura del 1513 A. Dürer (Il cavaliere, la morte e il diavolo) representa -ha recordado Marramao (1986, 171-183)- un príncipe que se aleja de la ciudad fortificada con torres y muros y, en compañía de horribles figuras, se encamina hacia tierras desconocidas. Con esto se representa el fin de la securitas, cuyo garante era el príncipe y la irrupción en la política de metus et indigentia. La historia desvela su pragmatismo tucidideano y la política descubre abiertamente el lenguaje del conflicto y de la guerra. En el declive de la virtud reunificadora de la ciudad, la ruptura de los modelos unilineales de la vida social sobrecarga lo ético-político con escisiones y disimetrías, cuya semántica comienza a individualizar las otras «posibilidades» de un horizonte de la experiencia política que ha visto cambiar sus propias relaciones con la soberanía.

Si con relación al tiempo como respecto al espacio -escribirá Hobbes en 1679 en Behemoth, o bien El parlamento largo- se pudiese hablar de altos y bajos, creo verdaderamente que la parte más alta del tiempo sería aquella comprendida entre el 1640 y el 1660. Quien efectivamente, desde aquellos años, como desde la montaña del diablo, hubiera mirado el mundo y observado las acciones de los hombres... habría podido tener un panorama de cada clase de injusticia y de cada tipo de locura que el mundo era capaz de ofrecer y también habría podido constatar como ésas eran producidas por sus madres: la hipocresía y la vanidad, de las cuales la una es doble iniquidad, la otra doble locura».

La injusticia y la locura que Hobbes entiende observables desde lo alto de la montaña del diablo están ahora totalmente alejadas de la felicidad pública que la ciudad podía asegurar. En ella, la antropología de la guerra está ligada a la inestabilidad y a la imprevisión de una comunicación en la cual prevalece -dice Hobbes- la duplicidad lingüística (más simplemente, en este 
caso, la hipocresía). Aquello que en el manierismo se estaba delineando como separación entre una comunicación pública y la «verdad» de la interacción privada -en la cual es todavía posible encontrar segmentos de certeza del intercambio social- ha ya caracterizado cada fragmento de la vida. Se ha perdido el sentido antagónico de conformidad-diversidad porque la incertidumbre ha penetrado también en la lógica de la interacción. Y Hobbes ha indicado el carácter ya efímero de una concepción de la política que no interiorice hasta el fondo la incertidumbre y la inestabilidad de los conflictos sociales. E1 «problema hobbesiano del orden», como dirá Parsons, se convierte en el problema de hallar una conexión funcional entre certeza e incertidumbre, estabilidad e inestabilidad, y en el asumir para el sistema de la política el mismo punto de vista de la «contingencia» de las relaciones de intercambio social a regular. Es en la teoría hobbesiana que la pacificación de los conflictos como obra de un soberano resulta explicada dentro de un gran proceso de mutación de la semántica política. La decisión política (die Entscheidung) necesita una nueva auto-nomía, una delimitación del «lugar del rey» diferenciado de todos los demás papeles sobre la base de un proceso de legitimación que provenga de un nuevo pacto de obediencia no más incontrolado.

También aquí es relevante la iconografía del soberano. Se manifiesta una progresiva pérdida de los caracteres antropomórficos del soberano mismo que, de makros anthropos, viene asumiendo la forma de horologium, machina, autómaton. Autómaton no en el sentido negativo habitualmente hoy atribuido, sino en el sentido clásico de mecanismo previsible, de ley capaz de contrastar y reducir el caso. En la lectura schmittiana es precisamente este carácter de previsibilidad del político lo que define la modernidad de la teoría de Hobbes. Mediante la diferenciación de la política de la sociedad, mediante su transformación en sistema particular junto a otros (N. Luhmann, 1983, 197), la aparente reducción de los espacios de la soberanía señala al contrario el máximo de expresión. Esta tiene lugar gracias a un cambio radical de los fundamentos de legitimidad del poder, lo que debe buscarse en el mandato proveniente de la sociedad civil. Si por consiguiente es un mecanismo político animado y controlado por la figura del soberano representativo para reducir el miedo individual y para consentir la «comunicación», la justificación del mandato necesita presuponer una ética de la renuncia de parte de cada uno de los individuos a cuotas de individualismo posesivo, pero de manera horizontalmente simétrica y dentro de una lógica de reciprocidad. Es así que la semántica 
de las relaciones políticas ve ampliar sus propios horizontes, saliendo de la tradicional clausura del vínculo soberano-súbdito.

La renuncia a cuotas de individualismo es vista en realidad como un proceso estratégico sugerido por la racionalidad comunicativa del taking role of the other, en la cual la imprevisibilidad de la doble contingencia viene simplificada gracias a mecanismos de autorreferencias. (N. Luhmann, 1983, 196.)

A partir de la gran teoría política del seiscientos, la justificación de este comportamiento estratégico acontece sobre la base de la elaboración del amor de sí: que no es paramétrico, ni es normatividad universalista, sino estrategia autorreferencial. La legitimidad del poder del soberano, sus propias chances de pacificación y hasta la autonomía de su propio ser ab-solutus descansan sobre el pactum a favor de la sociedad. En el propio seno de una política que habla el lenguaje de la guerra, la soberanía va elaborando conjuntamente un doble código de autonomía y de dependencia, de diferenciación y de «desdiferenciación», del uso de la violencia para evitar la violencia, sobre la base de un pacto simétrico y horizontal de la sociedad capaz de construir relaciones verticales representativas de una disparidad de poderes.

La consecuencia es que la vieja disimetría adscriptiva soberano-súbdito viene sustituida por una nueva disimetría adquisitiva producida voluntariamente -y racional por necesidad- por el pactum. Tanto en la versión iusnaturalista como en aquella utilitarista de la justificación de la soberanía, se abre camino una representación de la política que ya ha interiorizado la aparición de la complejidad de los temas y de las propias formas capaces, conjuntamente, de simetría y disimetría. Tal como en el manierismo político, también aquí se genera una alta cuota de complejidad por la producción de un surplus de cultura alrededor de la diferencia entre poder y obediencia, con juegos de inestabilidad en torno a la obligación política.

Desde la perspectiva de los súbditos (ex parte populi) esto significa que comienza a abrirse camino una «temporalización» de la política, y que el «manierismo» de la obediencia deja abierta una elección entre mundos posibles y contingencia de aquellas formas de gobierno de la sociedad. Es la convicción de la necesidad -revocable- de la renuncia a cuotas de individualismo en lugar de la seguridad que define identidad y diferencia de las dinámicas del poder. Su inestabilidad deriva de la continua reelaboración de las lógicas del amor de sí, que se auto-afirma como medio de una comunicación simbólicamente generalizada.

En lugar de ex parte principis, el convencimiento que surge alrededor de los peligros de la disimetría del propio poder respecto 
de los mandantes (Hobbes hablaba de una «turbulencia del hombre cuando más se encontraba a su agrado», 1974, 150) y la interiorización de los problemas de inestabilidad inducen a un importante proceso de desplazamiento semántico hacia la propia «juridificación». Y es así, tanto en el sentido de la monopolización del uso de la fuerza física (legitimación) como en un proceso histórico más largo: en la elaboración de una tendencia hacia la constitucionalización desde la óptica de un ensanchamiento de las bases racionales del consenso social, de la predecibilidad, etc. Y resulta significativo que sean introducidas, a partir de este momento, ciertas temáticas relativas a la continua producción del consenso en cuanto elemento del gobierno de la sociedad. Esto puede depender del hecho que convirtiéndose la propia inestabilidad en tema de autorreflexión para el sistema político, afecta a los mecanismos de turbulencia del ambiente como noises internos. $\mathrm{Y}$ la autorreflexión sobre sus propios mecanismos y la elaboración de estrategias de la propia identidad son ya un proceso estimulado de auto-referencia para el político. Una auto-referencia entendida como «diferenciación entre identidad y diferencia» es el vehículo de una modernización de la política que va recreando los propios elementos constitutivos, todo ello gracias a sí misma (N. Luhmann, 1985). Aun aumentando enormemente y cambiando de signo la racionalidad comunicativa entre los sistemas -la interpretación que de ella ha dado Polanyi aunque con otros lenguajes es significativa- se observa incrementar progresivamente la diferenciación de los sistemas mismos a través de clausuras auto-referenciales. Los primeros efectos evidentes de todo esto son, por una parte, aquello que habíamos definido como un deslizamiento semántico de los conceptos de un sistema a otro, según una lógica no arbitraria de reducción de la complejidad. Por otra parte, se revela una formalización interna en los sistemas de mecanismos que se aplican a sí mismos (mecanismos reflexivos) capaces de responder a problemas materiales y temporales de la complejidad del ambiente.

\section{Coniuratio}

Naturalmente, los dos procesos: a) deslizamiento semántico, b) aumento de la reflexividad, no sólo están interrelacionados sino que, desde muchos puntos de vista, representan un problema unitario.

Se trata de cuestiones que, desde diversos ángulos, han sido 
estudiadas profundamente por la Sozial Geschichte y por la teoría general de los sistemas sociales y cuyos resultados aquí únicamente podemos mencionar. La reflexividad se refiere a los medios de comunicación de los sistemas que son aplicados a sí mismos antes de ser aplicados al exterior (por ejemplo, el poder al poder; las decisiones a las decisiones; la regla a la regla; etc.), su aumento es una adquisición cualitativa de la auto-organización de un sistema (J. P. Dupuy, 1982) en términos de diferenciación de las propias funciones y de generalización de la comunicación; de forma que atiende a la resolución de problemas de «excedencias de posibilidades». De ese modo, cuando más generalizados son los mecanismos reflexivos tanto más su propia «redundancia» (H. Atlan, 1979) es capaz de neutralizar la «variedad» de los elementos. En términos más simples, cuando un sistema más se organiza sobre sí mismo tanto más puedo organizar mecanismos externos.

En el ámbito de la progresiva deformación de los elementos sociales -en la explosión de la variedad de los intereses, de los sujetos- la uniformidad no puede ser asegurada más que por una representación «banal» (trivial) del poder fundado sobre el ámbito del soberano, y ni siquiera por la presuposición de una única ética religiosa. Este problema está, por ejemplo, en la base tanto de la lectura weberiana de la racionalización del mundo como en la de N. Elías sobre Zivilisation del occidente europeo, al menos en relación a la monopolización de la fuerza física. Si ya a partir del manierismo, en efecto, se abre camino la idea que «el soberano no puede mandar a las conciencias», ello depende del hecho de que la diferenciación de los sistemas de acción requiere una «generalización» diferente -reflexiva- de los propios medios de comunicación hacia aquellos mecanismos capaces de contener, mejor que otros, más «diversidad», más excedencias. No es casual que los desplazamientos semánticos más significativos vayan desde la ética religiosa hacia el sistema político jurídico.

El concepto de tolerancia, por ejemplo, aparece todavía connotado religiosamente en el Shatesbury de la Lettera sull'entusiasmo y en Voltaire, y antes en cambio lo reencontramos como tema de discusión, o sea, formalmente conceptualizado, en el sistema político; cuando se hace evidente la crisis del absolutismo por una parte y se han multiplicado los sujetos sociales. No es casual que la tolerancia deba «juridificarse» para readuceuarse a los juegos de estabilización de la inestabilidad, transformándose en regla constituyente -en sentido técnico- para los sistemas constitucionales modernos. Su carácter constitutivo radica 
en el hecho que es condición de aquello que regula, como cuando se convierte en principio mayoritario en el sistema político estableciendo que la tutela de la minoría es confiada al mismo juego de la mayoría. El tránsito de la semántica religiosa de la tolerancia a la procedimentalización jurídica de las decisiones colectivas no considera exclusivamente las mutaciones de la idea de Communitas, sino también una articulación elevada del juego de la comunicación política en la cual la forma de la soberanía -y la estructura de su regla- son el círculo final y el efecto más visible. La incorporación en el sistema político del concepto de tolerancia sedimenta viejos significados y por eso experimenta nuevamente, inexplorados: la estructura moderna de la relación mayoría-minoría justificada como técnica de decisión colectiva y al mismo tiempo como principio «racional» (o menos irracional, E. Ruffini, 1976) de tutela de las minorías; internaliza dentro de la forma procedimental (simetría, medida) una disimetría, una desmesura del poder decisorio. Si en la ética religiosa que se va secularizando el reconocimiento de la diferencia surge gracias a un surplus de cultura de parte del tolerante respecto del tolerado (aun cuando es cálculo estratégico y no solamente «paramétrico»), en la actividad ético política el recurso a la tolerancia revela más abiertamente los juegos estratégicos del tolerante, cuando la estabilización temporal o social se convierte en un problema. A tal fin es ejemplar la lectura que Marx ha realizado en $L a$ Ideología Alemana de los procesos de universalización ideológica de la burguesía, en los cuales el momento culminante se configura con el reconocimiento de la igualdad formal de los ciudadanos en las grandes codificaciones burguesas. Cuando aún la herejía, según la expresión de Shaftesbury, no indicaba una simple opinión privada porque se usaba no sin «pasión» y señalaba aún un límite firme entre conformidad y disconformidad, la tolerancia obviamente no se constituía en tema de la comunicación. Serán las estrategias de diferenciación con el relativo aumento de los procesos auto-referenciales los que sugieren una solución manierística en la cual es la conformidad la que cubre la disformidad y luego la simetría la que incorpora disimetría.

Análogamente, en el sistema económico, la disimetría de las posiciones de intercambio debe encontrar justificación dentro de una armonía «natural de los intereses» -como en la fábula de las abejas de Mandeville- o bien en la hetero-regulación por parte de una «mano invisible» (Smith). La regla abiertamente utilitarista de una democracia -no solamente económica-, cuyo fin es la evidente desmesura de la «mayor felicidad del mayor número de ciudadanos», tiene como «destino» la igualdad formal 
del inter-cambio entre equivalentes bajo forma de contrato entre sujetos abstractos de Derecho; y es bien sabido que en los sistemas jurídicos modernos las relaciones de producción son simplemente contratos. Es una historia hasta demasiado repetida aquella que la necesidad de una gran producción de ideología nace para devolver solidez y uni-formidad a los sistemas sociales que viven la explosión y la multiplicación de las subjetividades con sus expectativas y con los respectivos horizontes de experiencia como problemas reales de la estabilidad (troublesome).

En la raíz de la modificación de esta antropología, primero manierista de conformidad -disconformidad, luego barroca de simetría-disimetría y después iluminista de igualdad-desigualdad, está la modificación de una representación compleja de la regla fundamental de la sociedad, de un nomos originario.

El tránsito descripto por Hobbes de una estructura antropomórfica a la idea de un soberano máquina individualiza uno de los puntos más decisivos de la modificación de la semántica de la legitimación. Por una parte, está el reconocimiento de la soberanía como una artificial reason creada por los mismos mecanismos del conflicto de la sociedad, gracias a una renuncia y a una delegación de cuotas de poder. La producción que autoriza la regla -antes se decía: una disimetría «necesaria»- nace, pues, de un pacto horizontal entre los asociados; y la consecuencia es que ya ha declinado la idea de una regla, válida desde siempre, que ha de ser obedecida (iusiurandum).

El derecho producido por esta razón artificial más que eliminar el arbitrio ab-solutum del soberano (autoritas non veritas facit legem) lo liga al pacto que constituye el poder delegado. No es una casualidad que haya sido conceptualizado a este respecto el derecho de resistencia que disuelve la obligación política en presencia del tirano. La semántica de la regla se desplaza de un derecho sobre el cual obedecer a un derecho para obedecer mediante un pacto, de un derecho paterno a un derecho fraterno (cum-iurandum) (A. M. Battista, 1966; G. Marramao, 1986). Es entonces la conjura (coniuratio) la que define la semántica de la política moderna, el jurar conjuntamente lleva dentro el sentido de una reducción «representativa» del soberano que, a través de la simetría del pacto entre iguales, evita para los asociados el destino del padre.

En el cuadro manierista del theatrum europaeum la conjura simboliza la escena fundamental (Urszene) del parricidio, a la búsqueda de una medida de la igualdad. La elaboración iluminista de la democracia y la idea de los sistemas jurídicos modernos de fundar la legitimidad sobre la legalidad constitucional (o 
del pacto constitucional) aun en sus resultados formalistas extremos, permanecen ancladas a esta escena fundamental que define el sentido de la teología política moderna.

El resurgir bajo otra forma -dentro de una semántica que transforma el sentido antagónico de sus conceptos- de disimetrías, desigualdades, inseguridades en el interior mismo, no a pesar del pacto entre iguales, representa la vanidad y vacuidad de aquel parricidio. El arbitrio del soberano reaparece en su absolutismo incontrolable, también en la representación más procesal de las reglas de la democracia. No se trata entonces de liberarse con un acto voluntario de decisión de aquello que Foucault llama la obsesión de la soberanía -creo que también esto forma parte de aquella escena del theatrum europaeum.

Tal vez se trata, aunque dicho sin arrogancia, de comprender con mucha auto-ironía hasta qué punto nuestros discursos «iluministas» sobre el problema del presente están constreñidos y determinados por el lenguaje de la tradición; o, por volver a Koselleck, cuanta sedimentación histórica existe en nuestros conceptos; o, mejor, en qué medida nuestro horizonte de expectativa está dependiendo del espacio de experiencia.

Concluyendo entonces, una filosofía «pública» que escoge permanecer confinada en la descripción microfísica de las desigualdades individuales, como si fueran incidentes aislados de la democracia para corregir con políticas singulares de public choices, no es únicamente inadecuada y no está a la altura de una teoría de la sociedad compleja, sino que es fundamentalmente ideológica. El paternalismo, por tanto, no es un problema simple de posibilidades y límites de un poder de interferencia en la esfera de otros sujetos, sino que es una importante metáfora del pacto.

(Trad. de Jorge Malem Seña) 


\section{BIBLIOGRAFÍA}

H. Atlan, Entre le Cristal et la Fumée, París, 1979.

A. M. Battista, Alle origini del pensiero politico libertino, Milano, 1966.

V. Camps, La imaginación ética, Barcelona, 1983.

E. Diciotti, «Paternalismo», en Materiali per una storia della cultura giuridica. XVI, 2, 1986, 557. J. P. Dupuy, Ordre et desordre, París, 1982.

G. Dworkin, Paternalism, in R. Sartorius (ed.), Paternalism, Minneapolis, 1983, 19-34.

N. Elías, La civiltà delle buone maniere, Bologna.

E. Havelock, Dike. La nascita della coscienza, Bari, 1981.

T. Hobbes, Behemoth, trad. it., Bari.

- Leviathan, trad. it. Bari, 1974, 150.

R. Koselleck, Futuro passato, Génova, 1986.

—, Critica illuministica e crisi della società borghese, Bologna, 1972.

N. Luhmann, Struttura della società e semantica, Bari, 1983.

-, Amore come passione, Bari, 1985.

C. Marramao, «L'ossessione della sovranità», en Effetto Foucault, a cura di P. A. Rovatti, Milano, 1986, 171-183.

K. Polanyi, La grande trasformazione, Torino, 1974.

J. Rawls, Teoria della giustizia, Milano, 1982.

F. Ruffini, Il principio maggioritario, Milano, 1976.

R. Sartorius, Paternalism, Minneapolis, 1983, Introd.

C. Schmitt, Scritti su Th. Hobbes, Milano, 1986.

R. Schnur, Individualismo e assolutismo, Milano, 1979, 14.

Shaftesbury, Lettera sull'entusiasmo, Milano.

L. Strauss, Persecution and the Art of Writing, Glencoe III, 1952. 